\title{
Unilateral pallidotomy in the treatment of cervical dystonia: a retrospective observational study
}

\author{
Shiro Horisawa, MD, Atsushi Fukui, MD, Kotaro Kohara, MD, Takakazu Kawamata, MD, PhD, and \\ Takaomi Taira, MD, PhD
}

Department of Neurosurgery, Neurological Institute, Tokyo Women's Medical University, Tokyo, Japan

OBJECTIVE The objective of this study was to assess the efficacy of unilateral pallidotomy in patients with asymmetrical cervical dystonia.

METHODS This study retrospectively included 25 consecutive patients with asymmetrical cervical dystonia refractory to botulinum toxin injections, who underwent unilateral pallidotomy between January 2015 and April 2017. Toronto Western Spasmodic Torticollis Rating Scale (TWSTRS) scores were evaluated preoperatively and 1 week, 3 months, and 6 months postoperatively. The clinical responses were defined as good responders, exhibiting $>50 \%$ improvement in the TWSTRS score at 6 months postsurgery, or poor responders, exhibiting $<50 \%$ improvement in TWSTRS scores at 6 months postsurgery.

RESULTS Twelve and 9 patients showed right- and left-side rotation, respectively; 1 and 3 patients had right- and leftside laterocollis, respectively. The mean age of onset and duration of the disease were $40.2 \pm 13.9$ and $8.9 \pm 10.9$ years, respectively. Mean TWSTRS scores were $38.4 \pm 12.6(p<0.001), 17.3 \pm 12.4(p<0.001), 19.5 \pm 13.4(p<0.001)$, and $20.0 \pm 14.7(p<0.001)$, preoperatively and 1 week, 3 months, and 6 months postoperatively, respectively. Fourteen patients $(56 \%$ ) demonstrated $>50 \%$ improvement in their TWSTRS total score (mean improvement of TWSTRS total score $=70.5 \%) 6$ months postsurgically. Furthermore, preoperative TWSTRS severity score was a prognostic factor (odds ratio $1.37,95 \%$ confidence interval $1.06-1.78, p=0.003$ ).

CONCLUSIONS These results suggest that unilateral pallidotomy is an acceptable treatment option for asymmetrical cervical dystonia. Further investigations with a larger number of cases and longer follow-up period are required to confirm these data.

https://thejns.org/doi/abs/10.3171/2019.9.JNS191202

KEYWORDS cervical dystonia; globus pallidus internus; pallidotomy; functional neurosurgery

$\mathrm{C}$ ERVICAL dystonia is associated with involuntary neck movements ${ }^{6}$ such as horizontal rotation, anteand retroflexion, and lateral bending. These actions require a complex combination of bilateral neck muscles. Currently, the surgical treatment of choice for cervical dystonia is deep brain stimulation (DBS) of the bilateral globus pallidus internus $(\mathrm{GPi})$. This procedure typically improves symptoms, which are measured using the Toronto Western Spasmodic Torticollis Rating Scale (TWSTRS), by $50 \%-60 \% .^{14,19,21-23,36}$ To date, treatment of cervical dystonia is believed to require bilateral intervention of the GPi; however, historical evidence suggests that unilateral ablative surgery of thalamic nuclei, including ablation of the GPi (pallidotomy), was the standard treatment for cer-

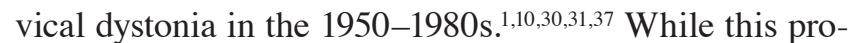
cedure typically demonstrated significant improvements in cervical dystonia, the lack of objective assessment scales, such as the TWSTRS or Burke-Fahn-Marsden Dystonia Rating Scale (BFMDRS), made this improvement difficult to quantify. Several case reports have demonstrated significant improvements in the symptoms of cervical dystonia following unilateral GPi-DBS. ${ }^{2,8,15,34}$ These studies indicate that unilateral pallidal intervention can improve cervical dystonia.

The evidence from the asymmetrical appearance of rotational (torticollis) or tilting (laterocollis) cervical dystonia has shown that the development of dystonia may be associated with asymmetrical brain abnormalities. ${ }^{26,28}$ This

ABBREVIATIONS BFMDRS = Burke-Fahn-Marsden Dystonia Rating Scale; DBS = deep brain stimulation; GPi = globus pallidus internus; SCM = sternocleidomastoid muscle; TWSTRS = Toronto Western Spasmodic Torticollis Rating Scale; VL = ventrolateral; VOi = ventro-oral intermediate.

SUBMITTED April 29, 2019. ACCEPTED September 25, 2019.

INCLUDE WHEN CITING Published online December 20, 2019; DOI: 10.3171/2019.9.JNS191202. 


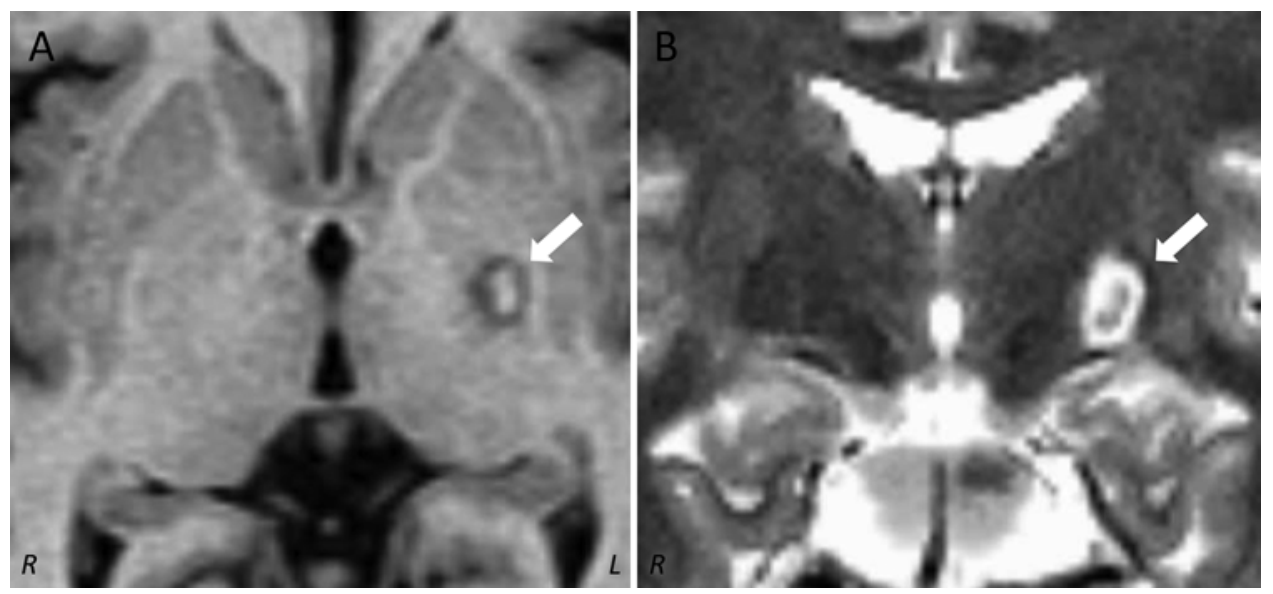

FIG. 1. Case 2. Postoperative axial T1- (A) and coronal T2-weighted (B) MR images showing a coagulated lesion (arrow) in the left GPi.

indicates that unilateral surgery on the symptomatic dominant hemisphere may be sufficient to improve asymmetrical cervical dystonia. In this study, we have retrospectively assessed the 6-month outcomes of unilateral pallidotomy for 25 consecutive patients with asymmetrical cervical dystonia (torticollis and laterocollis).

\section{Methods}

\section{Patient Population}

Twenty-five patients with rotational (torticollis) or tilting (laterocollis) cervical dystonia underwent unilateral pallidotomy at the Tokyo Women's Medical University Hospital between January 2015 and April 2017. All were refractory to botulinum toxin injections. The data for this study were retrospectively collected and analyzed. The ethics committee of Tokyo Women's Medical University approved this study, and considering the observational nature of the study, patient consent was waived. The primary research question was: does unilateral pallidotomy improve cervical dystonia?

\section{Evaluation Procedures}

TWSTRS scores were evaluated preoperatively and 1 week, 3 months, and 6 months postoperatively. The TWSTRS is a composite cervical dystonia scale consisting of subscales for severity, disability, and pain. ${ }^{4}$ The TWSTRS total score ranges from 0 to 85 and is the sum of the three subscores. The severity scale is a 10-item assessment of severity of cervical dystonia (range $0-35$, higher scores indicate greater severity). The disability scale is a 6-item assessment of activities of daily living (range 0-30, higher scores indicate greater disability). The pain scale is a 3-item assessment of pain severity, duration, and disability of cervical dystonia (range 0-20, higher score indicates greater pain). Head MRI scans were performed before and immediately after surgery, and 3 months postoperatively. Clinical responses were defined as follows: 1) good responders, exhibiting $>50 \%$ improvement in TWSTRS score 6 months postoperatively, and 2) poor responders, exhibiting $<50 \%$ improvement in TWSTRS scores 6 months postoperatively.

\section{Side of Surgery}

For torticollis, the contralateral hemisphere to the direction of head deviation was chosen as the surgical side, for example, right-side rotation-left pallidotomy or leftside rotation-right pallidotomy. For laterocollis, the contralateral side to the direction of neck tilting was chosen as the surgical side, for example, right laterocollis-left pallidotomy.

\section{Surgical Procedure}

The stereotactic target was a location posteroventrolateral to the GPi. The tentative target was a location 2-4 $\mathrm{mm}$ below, 20-22 $\mathrm{mm}$ lateral, and $2 \mathrm{~mm}$ anterior to the midpoint connecting the anterior and posterior commissures above the optic tract. A Leksell neurogenerator (Elekta) and monopolar radiofrequency probe $(1.0-\mathrm{mm}$-diameter tip with a 4.0-mm uninsulated length) were used for stimulation and coagulation. Surgery was performed under local anesthesia without microelectrode recordings, and the absence of capsular responses and visual phosphenes was verified. After confirming that macrostimulation (130 $\mathrm{Hz}, 100-\mu \mathrm{sec}$ pulse width, up to 8-10 mA) did not affect the internal capsule and optic tract, coagulation of the tentative target was conducted at $70^{\circ} \mathrm{C}$ for 30 seconds. The electrode was withdrawn in 2- and 4-mm increments to increase the lesion size, resulting in 3 contiguous lesions. Thereafter, 3 additional contiguous lesions were created posterolateral to the tentative target, resulting in a total of 6 lesions. MRI scans of the head were performed immediately after surgery to confirm the presence of lesions (Fig. 1). The volumes and placement of the lesions were calculated using Leksell GammaPlan. We measured distances from the center of the lesion created to the third ventricle wall (mediolateral), midcommissural point (anteroposterior), and the anterior-posterior commissure plane (dorsoventral).

\section{Statistical Analysis}

Data were nonparametric. The Wilcoxon signedrank test and Friedman test were used to compare total TWSTRS and subitem scores (severity, disability, and pain 
TABLE 1. Clinical characteristics of the patients

\begin{tabular}{cc}
\hline \multicolumn{1}{c}{ Variable } & Value \\
\hline No. of patients & 25 \\
\hline Sex & 13 \\
\hline M & 12 \\
\hline F & $40.2 \pm 13.9$ \\
\hline Mean age at onset \pm SD, yrs & $8.9 \pm 10.9$ \\
\hline Mean disease duration \pm SD, yrs & 16 \\
\hline Movement type & 9 \\
\hline Tonic & 13 \\
\hline Phasic & 12 \\
\hline Side of surgery & $17.4 \pm 6.0$ \\
\hline Rt & $14.9 \pm 6.0$ \\
\hline Lt & $6.0 \pm 5.7$ \\
\hline Mean preop TWSTRS scores \pm SD & $38.4 \pm 12.6$ \\
\hline Severity
\end{tabular}

scores) to their respective baseline scores. Bonferroni correction was used for multiple comparisons, and the level of statistical significance was set at $\mathrm{p}<0.01$.

Logistic regression was used to identify the prognostic factors for poor outcomes. Each variable of interest (movement type, age at onset, disease duration, TWSTRS pre-severity, TWSTRS pre-disability, TWSTRS pre-pain, lesion volume) was examined in a univariate analysis, and those that were significant at the 0.20 level were included in a multivariate model. A p value $<0.05$ was considered statistically significant. All statistical analyses were performed using JMP (version 13.0.0, SAS Institute).

This study is rated evidence class IV because of the absence of a nonsurgical group and the open-label design. Anonymized grouped data will be shared upon request from a qualified investigator.

\section{Results}

Patient demographics are shown in Table 1. Of the 25 patients included in this study, 12 and 9 exhibited rightand left-side rotation, respectively, and 1 and 3 exhibited right- and left-side laterocollis, respectively (Table 1). The mean age of onset and duration of disease were $40.2 \pm$ 13.9 and $8.9 \pm 10.9$ years, respectively (Table 1$)$. The mean TWSTRS total scores were $38.4 \pm 12.6$ preoperatively, 17.3 \pm 12.4 at 1 week postoperatively $(\mathrm{p}<0.001), 19.5 \pm 13.4$ at 3 months postoperatively ( $\mathrm{p}<0.001)$, and $20.0 \pm 14.7$ at 6 months postoperatively ( $<<0.001 ;$ Fig. 2D). Analysis of the subitem scores (severity, disability, and pain) showed a significant reduction in all three subscores at 1 week, 3 months, and 6 months postoperatively (Fig. 2A-C). The average volume of pallidotomy lesions was $58.5 \pm 25.1$ $\mathrm{mm}^{3}$. The average locations of the lesions in the three stereotactic planes are shown in Table 2. Ten patients (40\%) exhibited rapid symptomatic improvement 1 week after surgery, which persisted for 6 months irrespective of the type of dystonic movement (phasic or tonic type). Fourteen patients $(56 \%)$ demonstrated $>50 \%$ improvement in
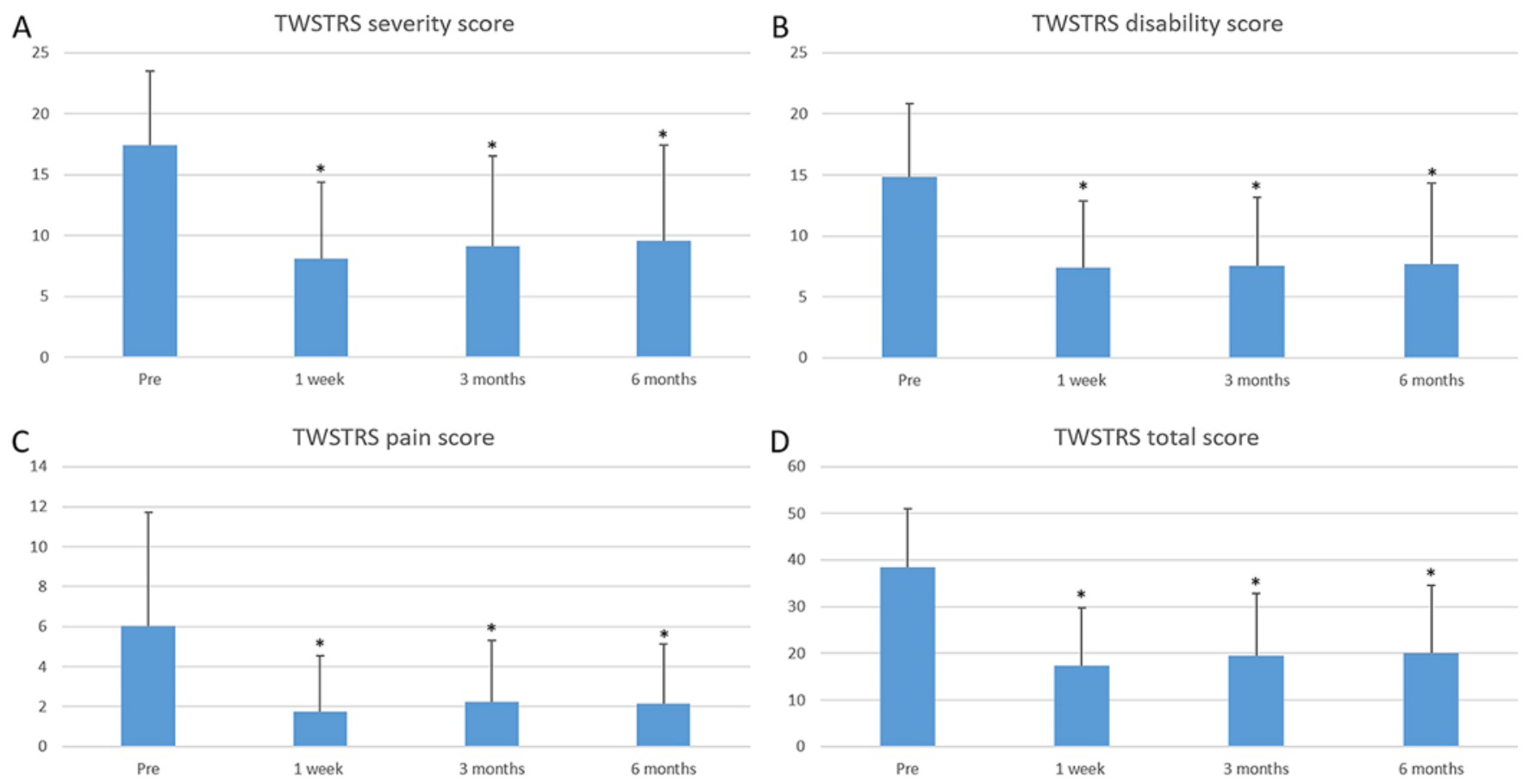

FIG. 2. TWSTRS scores before surgery and 1 week, 3 months, and 6 months after surgery. Mean $( \pm$ SD) scores for severity (A), disability (B), pain (C), and total score (D) on the TWSTRS before surgery and 1 week, 3 months, and 6 months after surgery. ${ }^{*} p<$ 0.0001 for comparison with the baseline score. Figure is available in color online only. 
TABLE 2. Summary of lesion volume and localization

\begin{tabular}{|c|c|}
\hline Lesion Variable & Mean \pm SD \\
\hline Lesion volume, $\mathrm{mm}^{3}$ & $58.5 \pm 25.1$ \\
\hline \multicolumn{2}{|l|}{ Lesion localization } \\
\hline \multicolumn{2}{|l|}{ Mediolateral plane, $\mathrm{mm}$} \\
\hline Distance from lesion to the 3rd ventricle wall & $17.2 \pm 1.6$ (lateral) \\
\hline \multicolumn{2}{|l|}{ Anteroposterior plane, $\mathrm{mm}$} \\
\hline $\begin{array}{l}\text { Distance from lesion to midcommissural } \\
\text { point }\end{array}$ & $2.4 \pm 1.7$ (anterior) \\
\hline \multicolumn{2}{|l|}{ Dorsoventral plane, $\mathrm{mm}$} \\
\hline $\begin{array}{l}\text { Distance from lesion to anterior-posterior } \\
\text { commissure plane }\end{array}$ & $2.3 \pm 0.9$ (ventral) \\
\hline
\end{tabular}

TWSTRS total score (mean improvement $70.5 \%$ ) at the 6-month follow-up evaluation and were defined as "good responders." Furthermore, preoperative TWSTRS severity was a prognostic factor (odds ratio $1.37,95 \%$ confidence interval 1.06-1.78, $\mathrm{p}=0.003$; Table 3). Eight (72.7\%) of the 11 patients in the "poor responders" group exhibited significant improvements at the 1-week follow-up evaluation; however, they experienced a recurrence of dystonic symptoms at the 6-month follow-up evaluation. Lesion volume and localization did not correlate with the outcomes.

One patient suffered from a cerebral infarction in the posterior limb of the right internal capsule next to the right pallidotomy lesion 1 month after surgery. This was considered a pallidotomy-related delayed stroke. The patient developed left-side hemiparesis and dysarthria, which resolved within 3 months after the onset of the infarction. No other perioperative complications were observed.

\section{Discussion}

The results of this study suggested that unilateral pallidotomy was associated with a mean improvement of $47.9 \%$ in total TWSTRS score, which is comparable to the results from bilateral GPi-DBS for cervical dystonia. ${ }^{14,19,21-23,36} \mathrm{In}$ agreement with our previous study of pallidotomy for dystonia, the effects of the procedure were visible within 1 week after surgery. ${ }^{11-13,20}$ In contrast, the effects of GPiDBS in patients with dystonia typically presented after several months. ${ }^{3,19,22}$

Several case reports have demonstrated significant improvements in cervical dystonia following unilateral pallidal surgery, including DBS and pallidotomy. Işlekel et al. have reported that right-side GPi-DBS resulted in considerable improvements in cervical dystonia with right-side rotation..$^{15}$ In addition, Escamilla-Sevilla et al. demonstrated that the same procedure significantly improved cervical dystonia with left-side rotation and retrocollis. ${ }^{8}$ Interestingly, right-side GPi-DBS showed greater improvements in the BFMDRS movement scale $(94.1 \%)$ when compared with the bilateral GPi-DBS (41.2\%) in this particular case. Torres et al. have reported that right-side GPi-DBS in a patient with cervical dystonia is characterized by rightside rotation and left-side bending, resulting in a $60 \%$ improvement in TWSTRS scores at 10 months postsurgically. ${ }^{34}$ Valálik et al. have reported unilateral pallidotomy
TABLE 3. Results of the logistic univariate and multivariate regression analyses relative to the risk of poor outcome 6 months after surgery

\begin{tabular}{lll}
\hline \multicolumn{1}{c}{ Risk Factor } & \multicolumn{1}{c}{ OR $(95 \% \mathrm{Cl})$} & $\mathrm{p} \mathrm{Value}$ \\
\hline Univariate analysis & & \\
\hline Movement type, phasic/tonic & $2.08(0.396-10.948)$ & 0.383 \\
\hline Age at onset & $0.992(0.94-1.05)$ & 0.7987 \\
\hline Disease duration & $1.09(0.97-1.21)$ & 0.0736 \\
\hline TWSTRS score pre-severity & $1.34(1.04-1.73)$ & 0.0076 \\
\hline TWSTRS score pre-disability & $1.08(0.84-1.39)$ & 0.5441 \\
\hline TWSTRS score pre-pain & $0.93(0.77-1.13)$ & 0.4694 \\
\hline Lesion volume & $1.01(0.98-1.05)$ & 0.3249 \\
\hline Multivariate analysis & & \\
\hline TWSTRS score pre-severity & $1.34(1.06-1.72)$ & 0.0030 \\
\hline Disease duration & $1.05(0.92-1.20)$ & 0.03879 \\
\hline
\end{tabular}

improved cervical dystonia and blepharospasm (Meige syndrome) for 2 years. ${ }^{35}$ These studies, concurrent with the results presented here, suggest that unilateral pallidal surgery may significantly improve cervical dystonia.

Between 1960 and 1980, before the introduction of selective peripheral denervation and DBS, unilateral lesioning surgery was the treatment of choice in patients with cervical dystonia because it avoids several severe complications associated with bilateral lesioning procedures. ${ }^{1,5,10,24,37}$ This has led to controversies regarding which surgical side provided better results, which remains unclear to date. The stereotactic targets for treatment of cervical dystonia include the ventrolateral (VL), ventrooral intermediate (VOi), and centro-median nuclei of the thalamus; the interstitial nucleus of Cajal; the zona incerta; and Forel's fields, H1 and H2. Cooper reported that unilateral VL thalamotomy was associated with a significant improvement in cervical dystonia with right-side rotation after 1 year, and this was accompanied by improvements in abnormal EMG activity demonstrated by the bilateral sternocleidomastoid muscle (SCM). ${ }^{5}$ Von Essen et al. have performed unilateral VOi thalamotomy in 17 patients diagnosed with cervical dystonia ${ }^{37}$ and reported a complete resolution in 4 patients, $75 \%$ improvement in 7 patients, $50 \%$ improvement in 3 patients, 25\% improvement in 2 patients, and no improvement in 1 patient. They placed the lesion in the VOi nucleus contralateral to the side toward which the face was turning in the horizontal component of the torticollis, which is consistent with our determination of surgical side. However, historical studies from 1960 through the 1980s did not use objective evaluation using scores, such as the TWSTRS and BFMDRS., ${ }^{1,5,10,24,37 \text { Fur- }}$ thermore, these studies did not use imaging evaluations of lesions using CT and MRI, which prevented any further understanding of the effects of unilateral lesioning surgeries in patients diagnosed with cervical dystonia.

Surgical treatment of cervical dystonia may be performed either on the side that is contralateral to the contracting SCM or on the side with the contracting muscle (ipsilateral surgery). Cooper employed the former method, while Hassler and Dieckmann, consistent with the current 
study, selected the latter., 5,8,10,15,24,34,37 Numerous studies have reported ipsilateral innervation of the SCM. Feline studies have reported that stimulation of the globus pallidus-entopeduncular nucleus (corresponding to the GPi in humans) increases neuronal firing rates of the ipsilateral SCM. ${ }^{17}$ Hassler and Dieckmann demonstrated that pallidothalamic stimulation increased the EMG activity in the ipsilateral $\mathrm{SCM},{ }^{10}$ while other studies reported SCM weakness caused by ipsilateral hemispheric strokes ${ }^{27}$ and injection of barbiturates into the unilateral carotid artery (as shown by the Wada test). ${ }^{7}$

The left SCM, right splenius, semispinalis, and trapezius muscles play a significant role in the rotation of the neck toward the right side. Generally, muscles receive only contralateral innervation from the unilateral hemisphere. If the SCM is exceptionally controlled by the ipsilateral hemisphere as mentioned above, neck rotation movement typically results from one hemisphere. Therefore, unilateral intervention might be sufficient in cases of rotational cervical dystonia. However, this hypothesis was discovered to be incorrect, because approximately half of the patients did not benefit from the unilateral pallidotomy. The symptomatic laterality is usually unequal in patients presenting with dystonia affecting multiple regions, such as blepharospasm with cervical dystonia (Meige syndrome). Valálik et al. have reported marked improvements associated with contralateral pallidotomy of the symptomatic dominant side in patients with Meige syndrome. ${ }^{35}$ Furthermore, our previous work showed that blepharospasms affecting the left side predominantly were completely resolved upon treatment with right pallidotomy. ${ }^{13}$

The evidence from these cases suggests that the development of dystonia may be associated with asymmetrical abnormalities, and surgery on the symptomatic dominant side increases the chances of observing satisfactory improvement in patients with cervical dystonia. Interhemispheric differences in pathological abnormalities have been reported previously in patients with cervical dystonia. For example, interhemispheric differences in local field potential oscillations, which reflect the local neuronal activity, in the GPi were observed in patients with torticollis and laterocollis, but not in those with retrocollis. ${ }^{26,28}$ Abnormalities in cortical excitability and intracortical inhibition are usually confirmed with transcranial magnetic stimulation in dystonia, and in rotational cervical dystonia they are lateralized in the hemisphere contralateral to the direction of head deviation, which implies that the hemisphere contralateral to the direction of head deviation may be a symptomatic dominant hemisphere. ${ }^{16}$ Intracranial structural lesions can lead to secondary cervical dystonia, while lesions in the unilateral basal ganglia may induce rotational cervical dystonia toward the side of head deviation. ${ }^{25,29,32}$ The hemisphere contralateral to the direction of head deviation (ipsilateral SCM contraction) may be related to the clinical asymmetrical presentation of torticollis or laterocollis. In the current study, 14 patients with torticollis and laterocollis demonstrated more than 50\% improvement in their TWSTRS scores following unilateral pallidotomy in the hemisphere contralateral to the direction of head deviation. Moreover, this improvement persisted for 6 months. Asymmetrical pallidal output may be associated with the onset of torticollis and laterocollis, and surgery on the symptomatic dominant hemisphere may improve dystonic symptoms significantly. Patients with torticollis or laterocollis refractory to unilateral pallidal surgery may exhibit greater bilateral hemispheric involvement of pathologic pallidal outflow, and unilateral pallidal surgery cannot redress imbalanced interhemispheric pallidal outflow.

The asymmetrical phenotype observed in patients with cervical dystonia accompanied by rotation or tilting suggests that unilateral intervention may produce satisfactory results. Surgery on the ipsilateral side of the contracting SCM (the side that is contralateral to the direction of head turning) can significantly improve rotational cervical dystonia, while tilting cervical dystonia can be improved by surgery on the side that the neck bends toward. Unfortunately, the manifestations of antero- or retrocollis cervical dystonia imply symmetrical hemispheric abnormalities, thus making it difficult to recommend an optimal surgical side. ${ }^{26}$

The results of the present study, consistent with previous evidence, suggest that bilateral pallidal surgeries are not always necessary to improve rotational or tilting cervical dystonia. Eleven patients with poor results in this study may be expected to gain further improvement with DBS using the subthalamic nucleus or GPi bilaterally. Several articles have reported the effectiveness of bilateral DBS after failed pallidotomy or disease progression., ${ }^{9} 18,33,38$ Fourteen patients who showed good results may develop recurrence or a deterioration of symptoms at a later stage. In those cases, additional surgery including contralateral pallidotomy or DBS may be necessary. We have suggested contralateral surgery to patients exhibiting poor results, or patients who are not satisfied with their improvements at the 6-month evaluation. There was a greater number of patients who were unwilling to have a mechanical device, or cannot afford to undergo DBS economically or geographically. For these patients, unilateral pallidotomy is a valuable alternative treatment option.

This study has several limitations. One is study design, which was retrospective, open-label, and noncontrolled. Another limitation is the relatively small sample size (n $=25$ ) and short follow-up period (6 months). To further assess the efficacy of unilateral pallidotomy for cervical dystonia, additional investigations are needed that overcome these limitations.

\section{Conclusions}

The present study suggests that unilateral pallidotomy is an acceptable treatment option for asymmetrical cervical dystonia. Further investigations with a larger number of cases and longer follow-up period are required to confirm these data.

\section{References}

1. Bertrand C, Molina-Negro P, Martinez SN: Combined stereotactic and peripheral surgical approach for spasmodic torticollis. Appl Neurophysiol 41:122-133, 1978

2. Chang JW, Choi JY, Lee BW, Kang UJ, Chung SS: Unilateral globus pallidus internus stimulation improves delayed 
onset post-traumatic cervical dystonia with an ipsilateral focal basal ganglia lesion. J Neurol Neurosurg Psychiatry 73:588-590, 2002

3. Chung M, Huh R: Different clinical course of pallidal deep brain stimulation for phasic- and tonic-type cervical dystonia. Acta Neurochir (Wien) 158:171-180, 2016

4. Consky E, Basinski A, Belle L, Ranawaya R, Lang A: The Toronto Western Spasmodic Torticollis Rating Scale (TWSTRS): assessment of validity and inter-rater reliability. Neurology 40:445, 1990 (Abstract)

5. Cooper IS: Effect of thalamic lesions upon torticollis. N Engl J Med 270:567-572, 1964

6. Dauer WT, Burke RE, Greene P, Fahn S: Current concepts on the clinical features, aetiology and management of idiopathic cervical dystonia. Brain 121:547-560, 1998

7. DeToledo JC, Dow R: Sternomastoid function during hemispheric suppression by amytal: insights into the inputs to the spinal accessory nerve nucleus. Mov Disord 13:809-812, 1998

8. Escamilla-Sevilla F, Mínguez-Castellanos A, Arjona-Morón V, Martín-Linares JM, Sánchez-Alvarez JC, Ortega-Morenoa A, et al: Unilateral pallidal stimulation for segmental cervical and truncal dystonia: which side? Mov Disord 17:1383-1385, 2002

9. Fonoff ET, Campos WK, Mandel M, Alho EJ, Teixeira MJ: Bilateral subthalamic nucleus stimulation for generalized dystonia after bilateral pallidotomy. Mov Disord 27:15591563,2012

10. Hassler R, Dieckmann G: Stereotactic treatment of different kinds of spasmodic torticollis. Confin Neurol 32:135-143, 1970

11. Horisawa S, Goto S, Takeda N, Takano Y, Kawamata T, Taira T: Pallidotomy for writer's cramp after failed thalamotomy. Stereotact Funct Neurosurg 94:129-133, 2016

12. Horisawa S, Goto S, Takeda N, Terashima H, Kawamata T, Taira T: Bilateral pallidotomy for cervical dystonia after failed selective peripheral denervation. World Neurosurg 89:728.e1-728.e4, 2016

13. Horisawa S, Kawamata T, Taira T: Unilateral pallidotomy for blepharospasm refractory to botulinum toxin injections. Eur J Neurol 24:e39-e40, 2017

14. Hung SW, Hamani C, Lozano AM, Poon YY, Piboolnurak P, Miyasaki JM, et al: Long-term outcome of bilateral pallidal deep brain stimulation for primary cervical dystonia. Neurology $68: 457-459,2007$

15. Işlekel S, Zileli M, Zileli B: Unilateral pallidal stimulation in cervical dystonia. Stereotact Funct Neurosurg 72:248-252, 1999

16. Kanovský P, Bares M, Streitová H, Klajblová H, Daniel P, Rektor I: Abnormalities of cortical excitability and cortical inhibition in cervical dystonia evidence from somatosensory evoked potentials and paired transcranial magnetic stimulation recordings. J Neurol 250:42-50, 2003

17. Kavaklis O, Shima F, Kato M, Fukui M: Ipsilateral pallidal control on the sternocleidomastoid muscle in cats: relationship to the side of thalamotomy for torticollis. Neurosurgery 30:724-731, 1992

18. Kim JP, Chang WS, Park YS, Chang JW: Bilateral globus pallidus internus deep brain stimulation for DYT1+ generalized dystonia with previously received bilateral thalamotomy and unilateral pallidotomy. Stereotact Funct Neurosurg 89:205-209, 2011

19. Kiss ZH, Doig-Beyaert K, Eliasziw M, Tsui J, Haffenden A, Suchowersky O: The Canadian multicentre study of deep brain stimulation for cervical dystonia. Brain 130:28792886, 2007

20. Kohara K, Taira T, Horisawa S, Hanada T, Kawamata T: [Bilateral pallidotomy for tardive dystonia: a case report.] No Shinkei Geka 45:971-976, 2017 (Japanese)
21. Krause M, Fogel W, Kloss M, Rasche D, Volkmann J, Tronnier V: Pallidal stimulation for dystonia. Neurosurgery 55:1361-1370, 2004

22. Krauss JK, Loher TJ, Pohle T, Weber S, Taub E, Bärlocher $\mathrm{CB}$, et al: Pallidal deep brain stimulation in patients with cervical dystonia and severe cervical dyskinesias with cervical myelopathy. J Neurol Neurosurg Psychiatry 72:249-256, 2002

23. Kroneberg D, Plettig P, Schneider GH, Kühn AA: Motor cortical plasticity relates to symptom severity and clinical benefit from deep brain stimulation in cervical dystonia. Neuromodulation 21:735-740, 2018

24. Laitinen L: Stereotaxic treatment of spasmodic torticollis. Acta Neurol Scand Suppl 39 (4 Suppl 4):231-236, 1963

25. LeDoux MS, Brady KA: Secondary cervical dystonia associated with structural lesions of the central nervous system. Mov Disord 18:60-69, 2003

26. Lee JR, Kiss ZH: Interhemispheric difference of pallidal local field potential activity in cervical dystonia. J Neurol Neurosurg Psychiatry 85:306-310, 2014

27. Mastaglia FL, Knezevic W, Thompson PD: Weakness of head turning in hemiplegia: a quantitative study. J Neurol Neurosurg Psychiatry 49:195-197, 1986

28. Moll CK, Galindo-Leon E, Sharott A, Gulberti A, Buhmann C, Koeppen JA, et al: Asymmetric pallidal neuronal activity in patients with cervical dystonia. Front Syst Neurosci 8:15, 2014

29. Münchau A, Mathen D, Cox T, Quinn NP, Marsden CD, Bhatia KP: Unilateral lesions of the globus pallidus: report of four patients presenting with focal or segmental dystonia. J Neurol Neurosurg Psychiatry 69:494-498, 2000

30. Mundinger F, Riechert T: [Results of stereotactic brain operations in extrapyramidal movement disorders on the basis of postoperative and long-term studies.] Dtsch $\mathbf{Z}$ Nervenheilkd 182:542-576, 1961 (German)

31. Mundinger F, Riechert T, Disselhoff J: Long-term results of stereotactic treatment of spasmodic torticollis. Confin Neurol 34:41-50, 1972

32. Naumann M, Becker G, Toyka KV, Supprian T, Reiners K: Lenticular nucleus lesion in idiopathic dystonia detected by transcranial sonography. Neurology 47:1284-1290, 1996

33. Novak KE, Nenonene EK, Bernstein LP, Vergenz S, Cozzens JW, Rezak M: Successful bilateral subthalamic nucleus stimulation for segmental dystonia after unilateral pallidotomy. Stereotact Funct Neurosurg 86:80-86, 2008

34. Torres CV, Moro E, Dostrovsky JO, Hutchison WD, Poon YY, Hodaie M: Unilateral pallidal deep brain stimulation in a patient with cervical dystonia and tremor. J Neurosurg 113:1230-1233, 2010

35. Valálik I, Jobbágy A, Bognár L, Csókay A: Effectiveness of unilateral pallidotomy for Meige syndrome confirmed by motion analysis. Stereotact Funct Neurosurg 89:157-161, 2011

36. Volkmann J, Mueller J, Deuschl G, Kühn AA, Krauss JK, Poewe W, et al: Pallidal neurostimulation in patients with medication-refractory cervical dystonia: a randomised, sham-controlled trial. Lancet Neurol 13:875-884, 2014

37. von Essen C, Augustinsson LE, Lindqvist G: VOI thalamotomy in spasmodic torticollis. Appl Neurophysiol 43:159-163, 1980

38. Waln O, Jankovic J: Bilateral globus pallidus internus deep brain stimulation after bilateral pallidotomy in a patient with generalized early-onset primary dystonia. Mov Disord 28:1162-1163, 2013

\section{Disclosures}

Shiro Horisawa received consulting fees from Boston Scientific. Takaomi Taira received consulting fees from St. Jude Medical Japan and speaking fees from Daiichi-Sankyo. 


\section{Author Contributions}

Conception and design: Horisawa, Taira. Acquisition of data: Horisawa, Fukui, Kohara, Taira. Analysis and interpretation of data: Horisawa, Fukui, Taira. Drafting the article: Horisawa.

Critically revising the article: Horisawa, Fukui. Reviewed submitted version of manuscript: Horisawa. Approved the final version of the manuscript on behalf of all authors: Horisawa. Statistical analysis: Horisawa, Fukui. Study supervision: Horisawa, Kawamata, Taira.

\section{Correspondence}

Shiro Horisawa: Neurological Institute, Tokyo Women's Medical University, Tokyo, Japan. neurosurgery21@yahoo.co.jp. 